\title{
Manufacturing Readiness Assessment for Evaluation of the Microarray Patch Industry: An Exploration of Barriers to Full-Scale Manufacturing
}

\section{Ben Creelman}

PATH

Collrane Frivold

PATH

Sierra Jessup

PATH

\section{Gene Saxon}

PATH

\section{Courtney Jarrahian ( $\square$ cjarrahian@path.org )}

PATH https://orcid.org/0000-0003-3046-6891

\section{Research Article}

Keywords: microneedle patch, scale-up, manufacturing barriers, quality control, critical quality attributes, production

Posted Date: April 13th, 2021

DOI: https://doi.org/10.21203/rs.3.rs-413740/v1

License: (9) (1) This work is licensed under a Creative Commons Attribution 4.0 International License. Read Full License 


\section{Abstract}

Microarray patch (MAP) technology is a promising new delivery technology for vaccines and pharmaceuticals. Yet due to several differing and novel production methods, barriers to full-scale manufacturing exist. PATH conducted a manufacturing readiness assessment and follow-up interviews to identify both the current manufacturing readiness of the industry as well as how readiness varies by developer type and MAP type. Follow-up interviews identified barriers the industry faces in reaching full manufacturing readiness, including the perceived regulatory and investment risk of manufacturing MAPs at scale due to quality requirements and control methods, uncertain sterility requirements, lack of standard production methods (especially around dissolvable MAP drying methods), and the lack of available contract manufacturing organizations with MAP manufacturing capabilities. A Regulatory Working Group has been established to identify and address critical quality issues specific to MAP manufacturing with the aim of providing developers insight into what will be expected for MAP product approvals. Standardizing MAP production equipment and automatic, visual quality control could reduce the overall risk to developers and contract manufacturing organizations in pursuing pilotscale manufacturing capabilities and ultimately lower barriers to the scale-up of full medical MAP product lines.

\section{Introduction}

Microarray patches (MAPs), also referred to as microneedle patches, offer an alternative to injectable methods of administering drugs and biologics [1]. A MAP contains tens to thousands of projections less than $1 \mathrm{~mm}$ long designed to puncture the stratum corneum that are either coated with or composed of a dry formulation. There are several MAP types in development, including solid-coated, dissolving, and hydrogel that aim to deliver a vaccine or drug to the dermis or epidermis. Organizations advancing pharmaceutical MAP technology include academic groups and biotechnology companies of varying sizes. The pharmaceutical industry has also expressed interest in MAPs. For instance, Merck \& Co., Inc., has announced a partnership with Vaxxas Inc. to develop a vaccine MAP [2], and Serum Institute of India has contributed material for MAP clinical studies for both measles-rubella and hepatitis $B$ vaccines.

At present, production-scale manufacturing facilities exist only for cosmetic MAP products [3]. MAPs for the delivery of drugs and vaccines are in either preclinical or clinical development, and commercial-scale manufacturing of pharmaceutical MAPs is still, on the whole, in its infancy. One drug-delivery MAP for zolmitriptan (for migraine headache), developed by Zosano Pharma, has completed a phase 3 study [4]. However, the US Food and Drug Administration recently requested additional data in response to the company's New Drug Application for its Qtrypta ${ }^{\mathrm{TM}}$ MAP for migraine treatment due to inconsistent drug exposure levels in study recipients who used MAPs from different lots of the product, a setback for achieving regulatory approval of the first pharmaceutical MAP product in the United States [5]. A phase 3 study for an abaloparatide MAP to treat osteoporosis from Radius Pharma is currently ongoing [6,7] Several phase 1 studies have also been completed for seasonal influenza vaccine MAPs [8-11].

Many pharmaceutical MAP developers are using manual, lab-scale MAP fabrication processes not suitable for mass production. Since MAP manufacturing methods are not standardized and are highly dependent on MAP design, formulation requirements, and anticipated use case, production-scale manufacturing will require 
development of custom equipment and novel processes. A recent partnership between Harro Höfliger, a leading MAP production equipment development firm, and Vaxxas is aimed at developing the first high-throughput aseptic production line for solid-coated vaccine MAPs [12]. Each line will have a targeted throughput of up to 5 million units per week. Employing a modular manufacturing approach with multiple production lines would facilitate production of tens of millions of units per week. These targets suggest that it could be feasible and economical to produce MAPs at scale. However, stringent regulatory and manufacturing requirements for these types of medical products without well-established precedents will be a hurdle to the scale-up of vaccine and drug MAPs.

MAPs have the potential to foster substantial gains in access and adherence to vaccines and essential medicines and to reduce burdens on strained health systems, particularly in low- and middle-income countries where there is a shortage of health workers trained to give injections. This technology has been identified by the Vaccine Innovation Prioritisation Strategy Alliance-a collaboration between the Gavi Secretariat, World Health Organization, Bill \& Melinda Gates Foundation, United Nations Children's Fund, and PATH-as a priority innovation for advancement of development, policy, and access [13]. PATH's MAP Center of Excellence was established as a key resource to provide leadership and guidance to the MAP technology field in advancing product development, understanding public health needs, reviewing manufacturing technologies, defining regulatory pathways, and demonstrating commercial viability [14]. A key focus area of the MAP Center of Excellence has been on manufacturing, and to this end, PATH co-hosted a three-day MAP Manufacturing Workshop in collaboration with Harro Höfliger at its facility in Germany in January 2020. The Center aims to identify gaps in manufacturing readiness and risks associated with MAP manufacturing to inform both strategy for maturation of manufacturing technologies and future investment opportunities. Although the needs of the MAP product development process are fairly well understood, production readiness for MAP manufacturing has not been formally evaluated. Therefore, PATH conducted a manufacturing readiness assessment to provide industry stakeholders with information on the status of MAP manufacturing, general barriers to manufacturing scale-up, and potential approaches for mitigating these issues to help inform future investment in and development of the industry. This paper outlines the results of that assessment.

\section{Methods}

Our assessment consisted of both an online survey as well as individual follow-up interviews for added depth of information to quantify manufacturing readiness. To determine individual and aggregate levels of manufacturing readiness in the pharmaceutical MAP industry, we distributed a survey in December 2019 to academic and commercial developers. Potential participants were identified from literature reviews, internet searches, and PATH's database on technology developers related to MAP innovations. We used a manufacturing readiness assessment toolkit or "deskbook" [15] developed by the United States Department of

Defense to develop the survey questions, which were modified as appropriate to ensure relevance to developers of MAP technology.

The survey was used to identify the manufacturing readiness level (MRL) of each developer across various manufacturing categories. The ten MRLs are defined as follows:

- Level 1: Basic manufacturing implications identified. 
- Level 2: Manufacturing concepts identified.

- Level 3: Manufacturing proof of concept developed.

- Level 4: Capability to produce the technology in a laboratory environment.

- Level 5: Capability to produce prototype components in a production relevant environment.

- Level 6: Capability to produce a prototype system or subsystem in a production relevant environment.

- Level 7: Capability to produce systems, subsystems, or components in a production representative environment.

- Level 8: Pilot line capability demonstrated; ready to begin low-rate initial production.

- Level 9: Low-rate production demonstrated; capability in place to begin full-rate production.

- Level 10: Full-rate production demonstrated and lean production practices in place [15].

In line with the Department of Defense toolkit, each question in our survey corresponded to a distinct MRL in one of the following nine manufacturing categories:

- Technology \& industrial base.

- Cost \& funding.

- Process capability \& control.

- Quality management.

- Manufacturing personnel.

- Manufacturing management.

For this survey and analysis, each category was represented by a series of questions representing progressively higher MRLs. To ensure the survey was manageable and to improve completion rates, we selected a total of 45 questions related to a subset of MRLs most relevant to MAP development status. For example, no questions related to an MRL of 10 in any category were asked because it is common knowledge that no developer is at that stage with MAP technology. During the analysis phase, we assigned each developer an MRL for each category equivalent to the highest-level question answered in the affirmative in that category. Afterwards, the results from all respondents were aggregated to ensure anonymity.

As a follow-up to the online survey, the study team conducted in-depth qualitative interviews in February and March of $\mathbf{2 0 2 0}$ with a subset of respondents. To help identify gaps in manufacturing readiness, interviews were focused on technical questions related to the developer's specific MAP technology, including questions designed to better understand the manufacturing categories where developers scored the lowest MRL. Other interview topics were related to future manufacturing scale-up plans, resources, and timelines for MAP production.

\section{Results}

We distributed the survey to $78 \mathrm{MAP}$ developer organizations, and 27 completed the survey (34.6\% response rate), representing 10 countries. The majority of respondents were commercial organizations (75\%), with the remainder from academic organizations. Respondents included developers working on solid-coated, dissolving, and hydrogel MAP subtypes. Of the respondents, $58 \%$ and $37 \%$ indicated that their most advanced MAP 
product was a dissolving MAP or solid-coated MAP, respectively, with one organization electing not to respond (Figure 1). Although some developers are advancing hydrogel MAPs, none of the respondents indicated that it was their most advanced MAP product.

The survey illustrated that manufacturing readiness varied among MAP developers and assessment categories, with MRLs ranging from manufacturing concept/proof-of-concept stages (MRLs 2 and 3) to demonstrated pilot line capability (MRL 8).

An aggregate summary of average MRL ratings and MAP-specific interpretations for each manufacturing category based on all developer responses is shown in Table 1. The Technology \& industrial base and Quality management categories tied for the highest average score (6.6) whereas the Cost \& funding category had the lowest average score (4.0).

Table 1 Average manufacturing readiness level for nine manufacturing categories: scores and interpretation

\begin{tabular}{|c|c|c|}
\hline $\begin{array}{l}\text { Manufacturing } \\
\text { category }\end{array}$ & $\begin{array}{l}\mathrm{N}=27 \\
\text { developers }\end{array}$ & Interpretation ${ }^{a}$ \\
\hline $\begin{array}{l}\text { Technology \& } \\
\text { industrial base }\end{array}$ & 6.6 & $\begin{array}{l}\text { Industrial capabilities in place to support manufacturing of prototype } \\
\text { devices in a production relevant environment. }\end{array}$ \\
\hline Design & 5.0 & $\begin{array}{l}\text { Preliminary design work in progress and able to support evaluation of } \\
\text { manufacturability. }\end{array}$ \\
\hline Cost \& funding & 4.0 & $\begin{array}{l}\text { Processes, materials, and designs can provide reasonable estimates, } \\
\text { including capital expenditures. }\end{array}$ \\
\hline Materials & 5.9 & $\begin{array}{l}\text { Preliminary material specifications are in place and supply chain capacity } \\
\text { is identified. }\end{array}$ \\
\hline $\begin{array}{l}\text { Process } \\
\text { capability \& } \\
\text { control }\end{array}$ & 4.9 & Pilot line processes are identified at the component level. \\
\hline $\begin{array}{l}\text { Quality } \\
\text { management }\end{array}$ & 6.6 & $\begin{array}{l}\text { Quality plan and system is in place. Inspection processes and acceptance } \\
\text { criteria are identified. }\end{array}$ \\
\hline $\begin{array}{l}\text { Manufacturing } \\
\text { personnel }\end{array}$ & 4.6 & $\begin{array}{l}\text { Manufacturing skill sets are identified, and production workforce } \\
\text { requirements evaluated. }\end{array}$ \\
\hline Facilities & 4.9 & $\begin{array}{l}\text { Manufacturing facilities are being evaluated and plans developed for } \\
\text { prototype production. }\end{array}$ \\
\hline $\begin{array}{l}\text { Manufacturing } \\
\text { management }\end{array}$ & 5.1 & $\begin{array}{l}\text { Manufacturing strategy is refined based on preferred design concept. } \\
\text { Make/buy evaluations initiated and include production considerations for } \\
\text { pilot line. }\end{array}$ \\
\hline
\end{tabular}

a Adapted from: Department of Defense: Manufacturing Readiness Level Criteria Matrix, version 2018. Available at: http://www.dodmrl.com/MRL_Matrix_V2018.xlsx. Accessed March 3, 2021.

A summary of all assessment responses (academic and commercial developers combined) is shown in box plot format in Figure 1. This figure demonstrates the distribution of manufacturer readiness levels for each category. Among all developers, the Design category and the Process capability \& contro/ category represent the 
largest quartile spread in manufacturing readiness, whereas on average, Technology \& industrial base and Quality management were reported to be the furthest advanced categories. Cost \& funding, notably, had the lowest reported average of all categories.

Figure 2 visualizes the difference in responses for the two MAP types reported as most advanced in our survey: solid-coated and dissolving. For all manufacturing categories except Materials, developers with solid-coated MAPs as their primary focus self-reported being less advanced on average than those focusing on dissolving MAPs. For both MAP types, developers reported similar interquartile ranges of MRLs for the Process capability \& control, Manufacturing personnel, and Manufacturing management categories.

The responses by developer type (academic vs. commercial) are shown in Figure 3. Commercial developers reported a more advanced mean MRL in every category. On average, academic developers reported larger quartile spreads in MRLs than commercial developers.

Follow-up interviews with developers explored further the results of the survey and identified areas in need of additional development, including quality requirements, quality control methods, sterility requirements, and aseptic manufacturing complexity. The interviews also highlighted a lack of contract manufacturing organizations (CMOs) well positioned to transition MAP technologies to pilot- and commercial-scale production levels.

\section{MAP manufacturing workshop}

The results of the survey informed the preparations for a three-day "MAP Manufacturing Workshop" PATH hosted in collaboration with Harro Höfliger at its facility in Germany in January 2020. In total, 75 individuals participated representing 37 organizations. The workshop brought together MAP developers and key stakeholders to share learnings about scaling MAP manufacturing from lab to production and to identify shared manufacturing challenges and risks. The workshop was also an opportunity to disseminate information related to manufacturing scale-up as well as inform future MAP Center of Excellence activities by identifying key manufacturing barriers and potential solutions.

During the workshop, unique challenges of MAP platform production were highlighted, which aligned with the results of our manufacturing readiness survey, including quality and regulatory issues (such as imaging-based, in-line quality control processes and the implications of aseptic production). Open-source information on solutions to common challenges, such as drying time and automatic visual quality control systems, were suggested as potential areas for future focus, as well as applicator/indicator technologies.

\section{Discussion}

Manufacturing readiness varied considerably among MAP developers, with MRLs ranging from early manufacturing concepts to advanced pilot line capability. Readiness also varied for different manufacturing categories, with developers generally reporting the highest MRLs in Technology \& industrial base, Quality management, and Materials categories, whereas the Cost \& funding, Manufacturing personnel, and Process capability \& control categories were generally in earlier stages. 
It is notable that although the few pharmaceutical MAP products that are closest to licensure are mainly of the solid-coated type, the average readiness for dissolving MAP technology was found to be either equal to, or more advanced than, solid-coated MAP technology in all manufacturing categories. This may have been due to the specific solid-coated MAP developers that responded to the survey.

The manufacturing gaps and barriers to scaled manufacturing discussed in follow-up interviews included quality issues, sterility requirements, and CMO availability. These barriers also align with feedback raised by stakeholders during the MAP Manufacturing Workshop.

\section{Quality requirements and quality control methods}

A major barrier to entry for MAP developers is that this product class is lacking precedent, with minimal guidance documentation available for the design, development, and testing of MAPs; therefore, developers need to defend their own performance requirements for each new product. Through the MAP Center of Excellence, PATH has partnered with Cardiff University in Wales to establish a Regulatory Working Group to facilitate collaboration among MAP developers, regulatory authorities, and public health stakeholders in order to define the MAP delivery system and identify critical quality attributes, develop standardized test methods, and evaluate sterility requirements for the technology class [16].

For high-speed production of MAPs, novel technological innovations are likely to be needed to facilitate nondestructive in-line quality control, such as automated visual inspection of micron-scale MAP projections.

\section{Sterility requirements and aseptic manufacturing}

Since MAP technology falls somewhere between transdermal patches, which can be produced in low-bioburden environments, and intradermal injection technologies, which must be produced aseptically, it is unclear what level of sterility assurance will be required by regulators for commercial MAP products. The three possible manufacturing routes for MAP products are aseptic manufacturing, low-bioburden manufacturing followed by terminal sterilization, and low-bioburden-only manufacturing. Since some active pharmaceutical ingredients in vaccines and biologics cannot withstand terminal sterilization, developers must decide whether to expend significant resources pursuing aseptic production or instead develop a lower-cost, low-bioburden process and risk being denied approval by regulatory authorities if they are unable to demonstrate an acceptable level of risk.

Safety risks associated with low-bioburden manufacturing center around the potential of a MAP to cause a local infection (adverse event), which could lead to complications such as systemic infection. Studies have shown that because MAPs physically disrupt the skin barrier, microorganisms can penetrate beyond the stratum corneum-but not the epidermis (whereas a 21-gauge hypodermic needle has been shown to allow this). This suggests that a local or systemic infection is highly unlikely from use of MAPs [17]. Due to the lack of clinical precedent, justifying the acceptability of non-aseptic production would require a risk-based evaluation/testing effort, but the end result may be substantial reductions in manufacturing costs. This topic is also the subject of review by the MAP Regulatory Working Group. However, most developers are taking the conservative approach of using aseptic manufacturing for MAPs that cannot be terminally sterilized. 
Two of the complexities associated with aseptic manufacturing are the sterility requirements for MAP component materials that must be introduced into the production line (e.g., formulations, molds, packaging) and more extensive sterile product monitoring requirements, both of which are anticipated to increase the manufacturing costs [18]. Thus, significant investment in manufacturing would likely be required to scale up MAP manufacturing for late-stage clinical trials and/or production manufacturing $[1,19]$.

The drying process is also complicated by potential aseptic requirements-particularly for dissolving MAPs. Compared with solid-coated MAPs that can be dried rapidly (due to their comparatively small liquid volume), the larger liquid volume of dissolving MAPs requires longer drying times to form the microprojections that encapsulate the active pharmaceutical ingredient. If the manufacturer uses a continuous production line to achieve high production volumes, the drying step could significantly increase the manufacturing floor space and number of isolators required to maintain an aseptic environment during drying as well as increase the risk of product loss as a result of line shutdowns. Most of the survey respondents we interviewed who were focusing on dissolving MAPs identified drying as a significant design challenge. Therefore, continued research in this area should be prioritized.

\section{Contract manufacturing organization availability}

For traditional injectable pharmaceutical packaging technologies, such as vials and prefilled syringes, there are numerous $\mathrm{CMO}$ s available to implement both pilot- and production-scale filling runs using standardized filling equipment designed for these delivery devices. This significantly decreases the capital requirements for developers when engaging in early development and testing efforts. However, during the developer interviews, respondents commented that there were very few, if any, CMOs capable of supporting clinical trials with production of MAPs at a level of quality consistent with good manufacturing practice guidelines. Several barriers impede CMOs from pursuing MAP manufacturing capabilities. First, there is a risk that CMOs may invest resources in a production line for a MAP type (e.g., solid-coated vs. dissolving) that is not prioritized by developers. In addition, since turnkey production equipment is not available for most MAP technologies, it is difficult for $\mathrm{CMO}$ s to design manufacturing lines without established development partners. Finally, installing a MAP manufacturing line requires significant time, space, and finances that could be used on more reliable product lines (such as vial filling or blow-fill-seal manufacturing). These requirements would be even higher if aseptic manufacturing is required.

These issues facing CMOs are the same as those facing individual MAP developers; thus, if CMOs are incentivized to develop MAP production capacity, it could defray high initial costs for developers and help accelerate progress.

\section{Limitations of the survey}

The data presented here reflect the manufacturing status of the MAP developers who completed the online survey, which is a portion of known MAP developers. The data are self-reported; as such, the MRLs generated by our analysis are based on the developers' perspectives and may not reflect their actual status. During the follow-up interviews, efforts were made to validate the online survey responses.

\section{Conclusion}


The PATH MAP Center of Excellence manufacturing readiness assessment survey revealed both the selfreported manufacturing readiness of the pharmaceutical MAP developer industry as a whole as well as how it varied between developer types and the prioritized MAP types. Follow-up interviews highlighted key barriers to full production-scale manufacturing that developers face, namely the perceived regulatory and investment risk of manufacturing MAPs at scale due to quality control requirements and methods, uncertain sterility requirements, lack of established large-scale production methods (especially around dissolvable MAP drying), and the lack of availability of CMOs with MAP capabilities. The MAP Regulatory Working Group is working to identify and address key issues specific to developing MAP manufacturing capabilities with the aim of providing developers insight into what will be expected for MAP product approvals. Technological advancements in MAP production equipment and automatic visual quality control could benefit many developers within this divergent technology class by enabling more $\mathrm{CMO}$ s to support pilot-scale manufacturing, ultimately lowering the barriers to full scale-up of medical MAP production lines.

\section{Declarations}

Ethics approval and consent to participate: These activities were determined to be not human subjects research (RDC\#1241) by the Research Determination Committee of PATH's Office of Research Affairs. Participants opted in and there was no requirement for formal written consent.

Consent for publication: All authors have reviewed and consented to publication, and the manuscript was approved by the organizational program leader. While participants were aware that the resulting data could be used for reports and/or publications, these activities were determined to be non-research and therefore no consent to publish was required.

Availability of data and materials: Raw data will not be made available due to its confidential nature.

Funding: This project was funded with UK aid from the UK government through the Foreign Commonwealth and Development Office.

Authors' contributions: GS, BC, and CJ conceived of the idea. BC, CF, and SJ designed and implemented the assessment. All authors contributed to the analysis of results and writing and review of the paper.

Acknowledgements: This project was funded with UK aid from the UK government through the Foreign Commonwealth and Development Office.

Conflicts of interest/Competing interests: The authors declare no competing interests.

Code availability: Not applicable.

\section{References}

1. Prausnitz M. Engineering microneedle patches for vaccination and drug delivery to skin. Annu Rev Chem Biomol Eng. 2017;8:177-200. https://doi.org/10.1146/annurev-chembioeng-060816-101514.

2. Business Wire. Vaxxas Announces That MSD Exercises Option to Apply Novel Immune System Activation Platform for Vaccine Candidate. 
https://www.businesswire.com/news/home/20200528005297/en/Vaxxas-Announces-MSD-ExercisesOption-Apply-Immune. Accessed March 3, 2021.

3. McCrudden MTC, McAlister E, Courtenay AJ, González-Vázquez P, Singh TRR, Donnelly RF. Microneedle applications in improving skin appearance. Exp Dermatol. 2015;24(8):561-6.

https://doi.org/10.1111/exd.12723.

4. Spierings EL, Brandes JL, Kudrow DB, Weintraub J, Schmidt PC, Kellerman DJ, Tepper SJ. Randomized, double-blind, placebo-controlled, parallel-group, multi-center study of the safety and efficacy of ADAM zolmitriptan for the acute treatment of migraine. Cephalalgia. 2018;38(2):215-24. https://doi.org/10.1177/0333102417737765.

5. Pharma Z. Zosano Pharma Receives Complete Response Letter from FDA for Qtrypta ${ }^{T M}$. https://ir.zosanopharma.com/news-releases/news-release-details/zosano-pharma-receives-completeresponse-letter-fda-qtryptatm. Accessed March 3, 2021.

6. Radius. Radius Health: Enrollment Completed for Phase 3 wearABLe trial. https://ir.radiuspharm.com/news-releases/news-release-details/radius-health-enrollment-completed-phase3-wearable-trial. Accessed March 3, 2021.

7. ClinicalTrials.gov. Phase 2 Study of BA058 (Abaloparatide) Transdermal Delivery in Postmenopausal Women With Osteoporosis. https://clinicaltrials.gov/ct2/show/NCT01674621. Accessed March 3, 2021.

8. Forster AH, Witham K, Depelsenaire ACl, Veitch M, Wells JW, Wheatley A, Pryor M, Lickliter JD, Francis B, Rockman S, Bodle J, Treasure P, Hickling J, Fernando GJP. PLoS Med. 2020;17(3):e1003024. https://doi.org/10.1371/journal.pmed.1003024.

9. Fernando GJP, Hickling J, Jayashi Flores CM, Griffin P, Anderson CD, Skinner SR, Davies C, Witham K, Pryor M, Bodle J, Rockman S, Frazer IH, Forster AH. Safety, tolerability, acceptability and immunogenicity of an influenza vaccine delivered to human skin by a novel high-density microprojection array patch (Nanopatch $^{\text {TM }}$ ). Vaccine. 2018;36(26):3779-88. https://doi.org/10.1016/j.vaccine.2018.05.053.

10. Rouphael NG, Paine M, Mosley R, Henry S, McAllister DV, Kalluri H, Pewin W, Frew PM, Yu T, Thornburg NJ, Kabbani S, Lai L, Vassilieva EV, Skountzou I, Compans RW, Mulligan MJ, Prausnitz MR, TIV-MNP 2015 Study Group. The safety, immunogenicity, and acceptability of inactivated influenza vaccine delivered by microneedle patch (TIV-MNP 2015): a randomised, partly blinded, placebo-controlled, phase 1 trial. Lancet. 2017;390(10095):649-58. https://doi.org/10.1016/s0140-6736(17)30575-5.

11. Hirobe S, Azukizawa H, Hanafusa T, Matsuo K, Quan YS, Kamiyama F, Katayama I, Okada N, Nakagawa S. Clinical study and stability assessment of a novel transcutaneous influenza vaccination using a dissolving microneedle patch. Biomaterials. 2015;57:50-8. https://doi.org/10.1016/j.biomaterials.2015.04.007.

12. Business Wire. Harro Höfliger and Vaxxas Announce Alliance to Develop Industrial-scale Aseptic Processing Line for Vaccine Products Based on Vaxxas' High Density Microarray Patch (HD-MAP). https://www.businesswire.com/news/home/20200528005295/en/Harro-H\%C3\%B6fliger-VaxxasAnnounce-Alliance-Develop-Industrial-scale. Accessed March 3, 2021.

13. Gavi. the Vaccine Alliance: The Vaccine Innovation Prioritisation Strategy (VIPS). https://www.gavi.org/ouralliance/market-shaping/vaccine-innovation-prioritisation-strategy. Accessed March 3, 2021.

14. PATH. The PATH Center of Excellence for Microarray Patch Technology. https://www.path.org/resources/path-center-excellence-microarray-patch-technology/. Accessed March 3, 
2021.

15. Department of Defense Instruction. Manufacturing Readiness Level (MRL) Deskbook. http://www.dodmrl.com/MRL\%20Deskbook\%20V2020.pdf. Accessed March 3, 2021.

16. Cardiff University PATH. Microneedle Array Patch Regulatory Working Group. https://www.microneedleregulatory.org/. Accessed March 3, 2021.

17. McCrudden MT, McAlister E, Courtenay AJ, González-Vázquez P, Singh TR, Donnelly RF. Microneedle applications in improving skin appearance. Exp Dermatol. 2015;24(8):561-6. https://doi.org/10.1111/exd.12723.

18. European Union. European Commission Good Manufacturing Practices Guideline, Annex 1. https://ec.europa.eu/health/sites/health/files/files/gmp/2017_12_pc_annex1_consultation_document.pdf. Accessed March 3, 2021.

19. Peyraud N, Zehrung D, Jarrahian C, Frivold C, Orubu T, Giersing B. Potential use of microarray patches for vaccine delivery in low- and middle- income countries. Vaccine. 2019;37(32):4427-34. https://doi.org/10.1016/j.vaccine.2019.03.035.

\section{Figures}

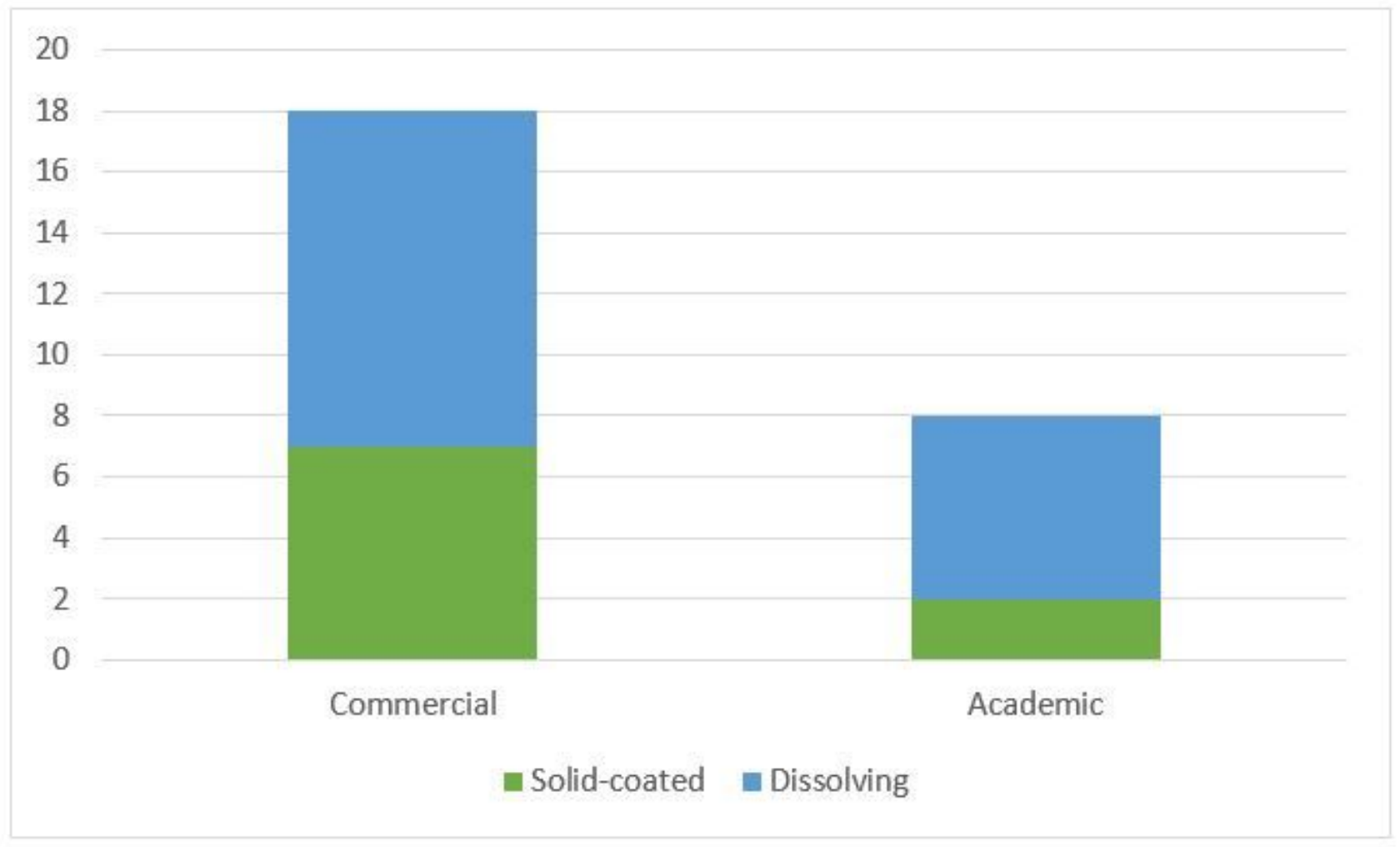

\section{Figure 1}

Most advanced microarray patch technology by type of developer* * Note: One developer did not answer this question and was not included in this figure. 


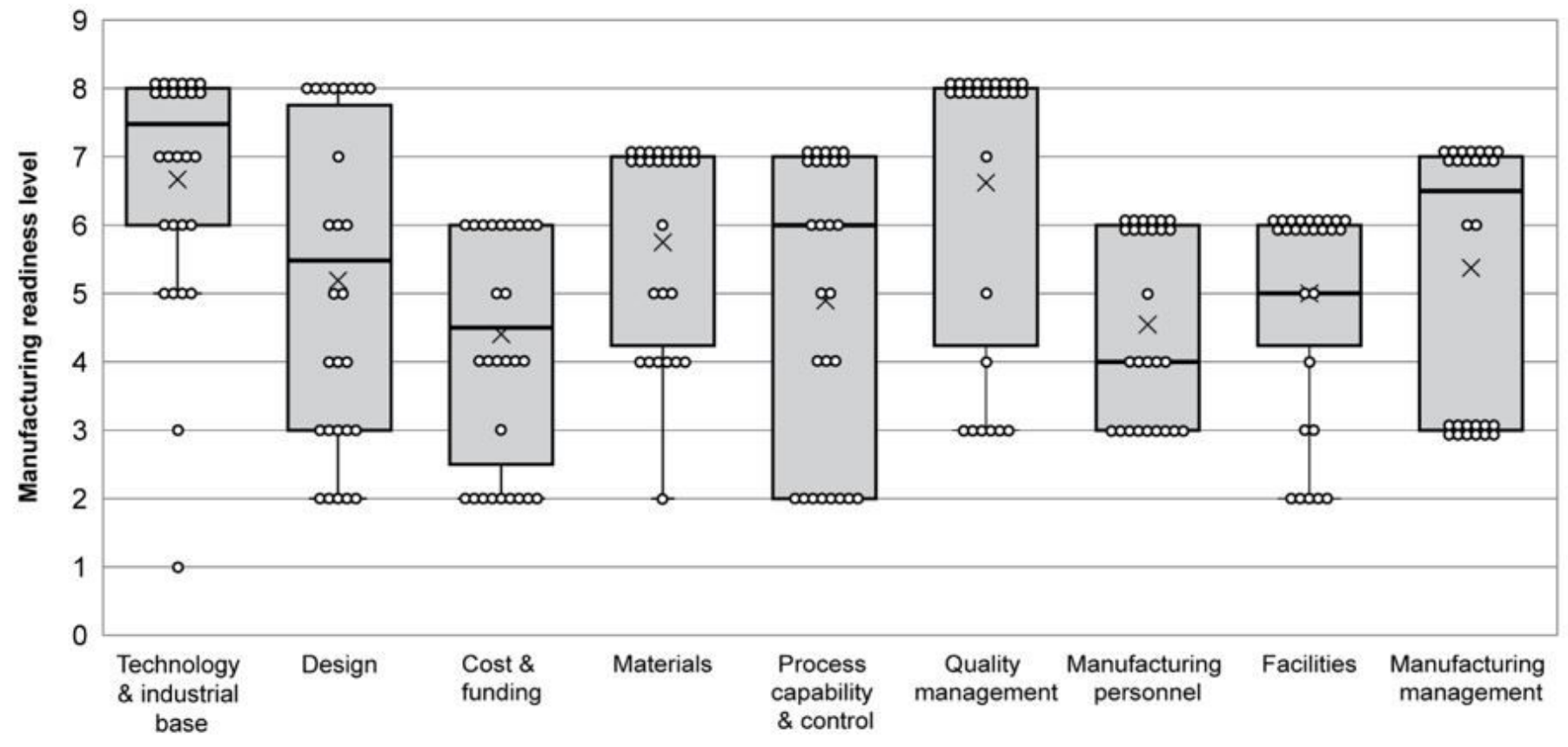

Manufacturing categories

O Individual response $\quad \times$ Mean

Figure 2

Summary of manufacturing readiness assessment results from all respondents $(N=27)$

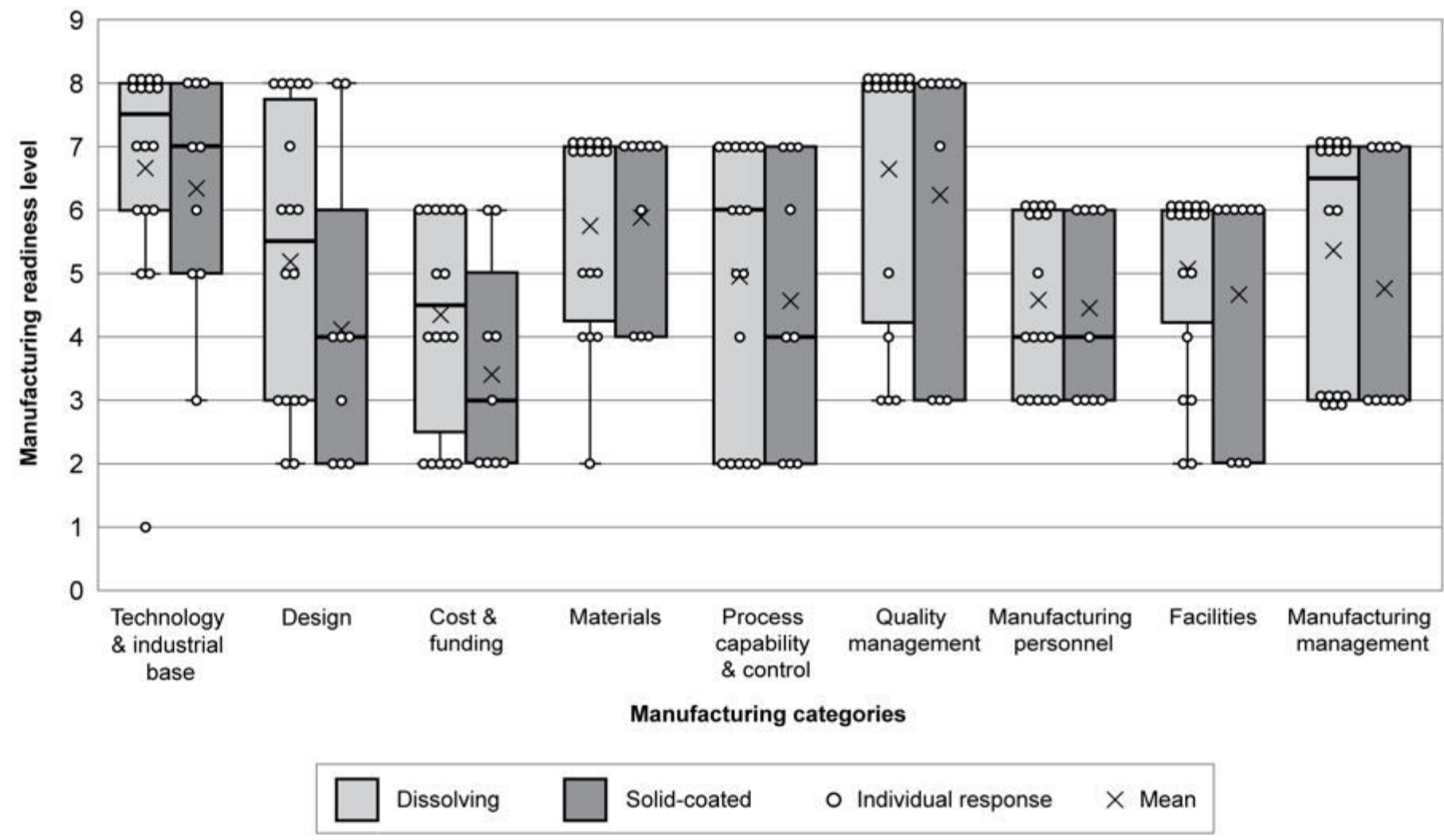


Figure 3

Manufacturing readiness assessment results for solid-coated vs. dissolving microarray patches. $(\mathrm{N}=27)$

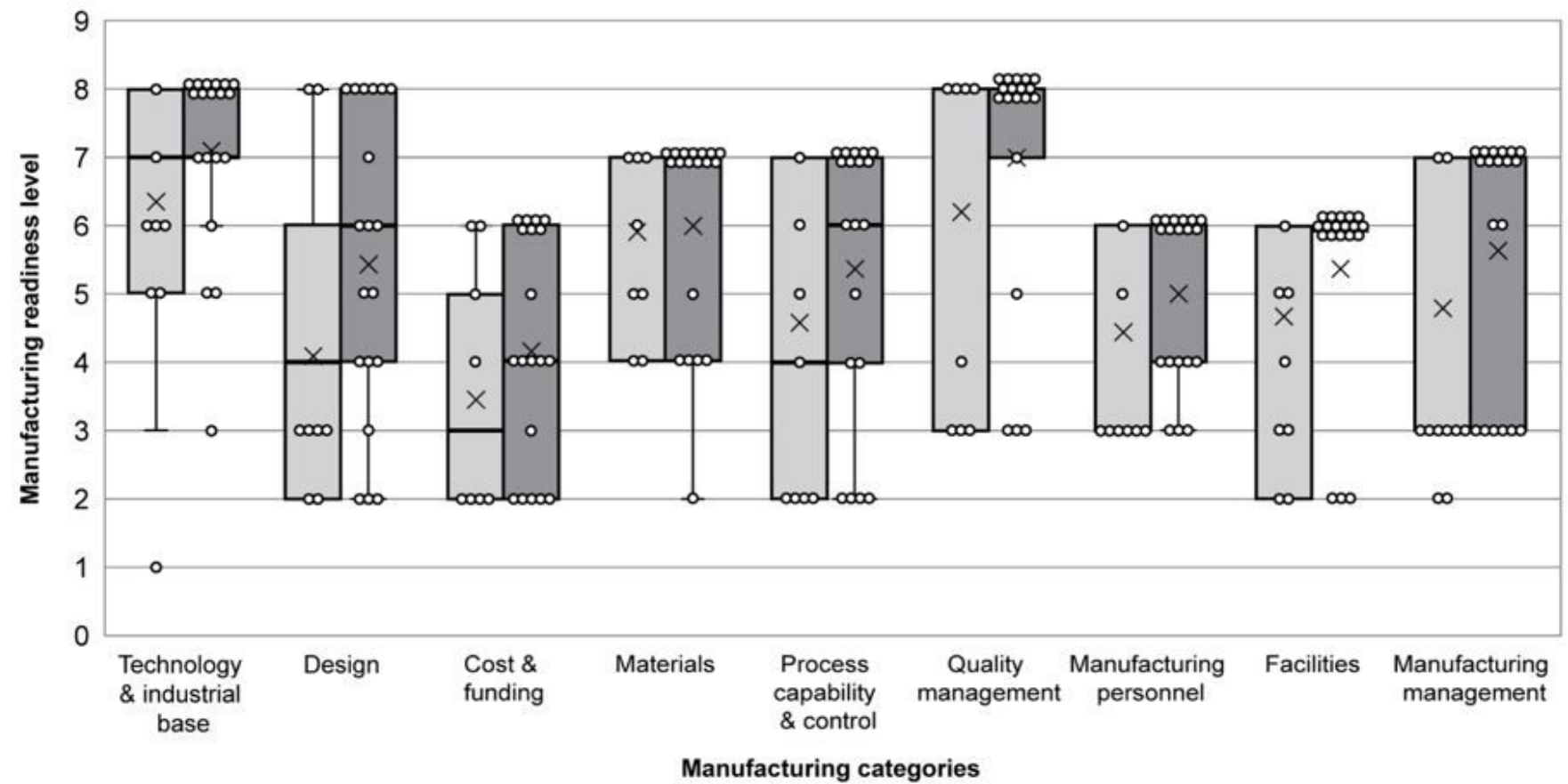

$\square$ Academic $\square$ Commercial $\quad 0$ Individual response $\times$ Mean

Figure 4

Manufacturing readiness assessment results per microarray patch developer type $(N=27)$

\section{Supplementary Files}

This is a list of supplementary files associated with this preprint. Click to download.

- GraphicalAbstract.jpg 